\title{
Trabajo sexual femenino en la ciudad de San José, Costa Rica: un enfoque sociobiológico al iniciarse el siglo XXI
}

\author{
Julián Monge-Nájera, Rosberly Rojas Campos, Roxana Morales Bonilla e Iris Amalia Ramírez S. \\ Universidad Estatal a Distancia, 474 - 2050 San Pedro Montes de Oca, San José, Costa Rica; julianmonge@gmail.com; rrojas@uned.ac.cr; \\ rmorales@uned.ac.cr; iramirez@uned.ac.cr
}

Recibido 3-VI-2009 Corregido 1-IV-2009 Aceptado 7-V-2009

\begin{abstract}
Female sex work in San Jose, Costa Rica: a sociobiology approach. In Costa Rica, sex work has been studied for decades from a pre-evolutionary perspective, and often with an underlying patriarchal bias because sex work is seen as a social pathology, ignoring contributions from sociobiology and evolutionary psychology. In this study, we measured the performance of predictions made by the evolutionary model, in a sample of 78 women aged 18 to 81 years, who perform sex work in San José, capitol of Costa Rica, interviewed in 2008. The interviews were based on a questionnaire, respondents were paid for their time, and data were analyzed with the SPSS statistical program. All predictions of the evolutionary; model are met by this sample: the customers are mostly men in reproductive age (29 to 54 years) who pay women for sexual activity even in cases in which they establish an emotional relationship with the workers. The predictions of the model for workers are also met, since they are primarily women of reproductive age ( 24 to 41 years), 100\% charge for their services, and women who are more attractive for their beauty, intellect and education, earn 10 times more than the rest. In the human species, resources can be given in exchange for sexual services, and $98 \%$ of these sex workers are women who have no other source of income; besides, $69 \%$ have no partner helping them to support their children. The other $31 \%$ have a partner but do not receive enough money from him.
\end{abstract}

KEY WORDS

Evolution of human sexual behavior, sex work, Costa Rica, sociobiology, evolutionary psychology, prostitution, sex for resources, patriarchy.

En las antiguas culturas de Irak, Egipto y Roma, el trabajo sexual fue un acto de piedad religiosa, como lo es aún en algunos lugares de la India (Murphy 1998). Sin embargo, esos casos son la excepción que confirma la regla, debido a que la mayoría de las sociedades han seguido un modelo fuertemente patriarcal (Schiebinger 1993). Para el patriarcado, la sexualidad humana es un fenómeno sucio y malo, que únicamente se tolera con fines reproductivos y dentro del matrimonio formal (Leigh 2008). Así, al transgredir la trabajadora sexual la ideología patriarcal, suele encontrarse en la parte más baja del escalafón porque enfrenta el doble estigma de ser mujer y de ser "prostituta" (Rojas y Scott 1994).

Ese rechazo social, que puede tomar forma de grave violencia física y sicológica (Murphy 1998), lleva a estas mujeres a vivir un fuerte conflicto personal, entre los valores patriarcales que han aprendido de fuentes religiosas desde niñas y su realidad inmediata como trabajadoras sexuales (Méndez 1992, Rojas y Scott 1994).

Según Darwin, Homo sapiens es una especie animal sujeta al proceso de evolución orgánica igual que todas las demás y por lo tanto sus sociedades también tienen fundamentos biológicos (Barkow 2005). En años recientes, la ciencia de los países industrializados ha entrado a una revolución evolucionista en el estudio del trabajo sexual y otros fenómenos de la sociedad humana (Barkow 2005). El origen de esta revolución incluye cierta insatisfacción con los resultados de la economía, la antropología y la sociología tradicionales, que han estado fundamentadas en la negación de que el ser humano sea un animal más (Bouissac 2006, Saad 2007).

A diferencia de la sociología pre-evolucionista, la 
sociobiología da explicaciones que son válidas tanto para la especie humana como para las demás especies animales. El intercambio de actividad sexual por recursos es una práctica que evolucionó en diversos organismos, incluyendo al grupo de los chimpancés al que pertenece Homo sapiens. En organismos tan diferentes como las moscas y los bonobos, los machos ofrecen alimento $u$ otros recursos a las hembras a cambio de relaciones sexuales (Geary 2006). El trabajo sexual es la versión humana de este fenómeno biológico en el cual las mujeres obtienen recursos, como dinero e influencia, a cambio de servicios sexuales (Geary 2006).

Al analizar la sociedad humana como un tipo particular de sociedad animal, la sociobiología y la sicología evolutiva incorporan un modelo de selección natural que complementa, ubica y filtra lo descubierto por las ciencias sociales pre-evolucionistas (Foster et al. 2007, Wilson y Wilson 2007, Buss 2008). La aplicación de principios biológicos al ser humano ha sido rechazada por las feministas de derecha como Farley (2005), la cuales se han sumado a los grupos ultraconservadores cristianos que también rechazan la visión biológica del ser humano (ambos grupos buscan expandir la visión de trabajadoras y clientes como delincuentes; Leigh 2008).

En contraste, las feministas que aceptan ampliamente la naturaleza sexual humana han visto el trabajo sexual como un derecho y un asunto laboral en el cual las mujeres obtienen poder (Leigh 2008).

Dentro del campo científico, la visión evolucionista ha sido paulatinamente incorporada en la sicología y la sociología de países industrializados (Wilson y Wilson 2007), no así en América Latina, donde se maneja aún un discurso pre-evolucionista. Sin embargo, la visión pre-evolucionista no puede explicar muchos fenómenos (Miller et al. 2007). Por ejemplo, las bailarinas exóticas de un estudio hecho en los EEUU ganaron US\$335 en propinas cuando estaban en estro, US\$260 en el periodo luteal y US\$185 durante la menstruación, por turno de 5 horas. El grupo "control" constituido por bailarinas que usaban píldoras anticonceptivas y no tenían el ciclo hormonal, ganaron montos similares a lo largo de todo el mes (Miller et al. 2007). Estos resultados son coherentes con el modelo evolucionista, que en años recientes ha sido apoyado en estudios basados en entrevistas, experimentos sociales, estudios morfométricos, experimentos fisiológicos y análisis de ADN (Alcock 2001, Wilson y Wilson 2007).

En Costa Rica, el trabajo sexual ha sido estudiado durante décadas según una visión pre-evolucionista. En los decenios de 1950 a 1970, se hicieron fundamentalmente estudios demográficos, que buscaban ubicar a estas trabajadoras en el marco de su relación con las enfermedades de transmisión sexual, y se buscaba "corregir su sentido moral" (López 1992, Rojas y Scott 1994).

Desde la década de 1980, los estudios ampliaron su temática y se agregó el aporte de mujeres investigadoras, quienes complementaban con sus puntos de vista femeninos el tradicional enfoque masculino que hasta entonces había dominado los estudios sobre este tema (ver bibliografía en Herrera 2000). Sin embargo, la mayoría de las investigaciones se centraron nuevamente en las trabajadoras sexuales como posibles "agentes dispersoras" de enfermedades, en este caso debido a la aparición del virus del SIDA (López 1992, Rojas y Scott 1994).

La etiqueta de la trabajadora sexual como tentadora que induce al hombre al pecado (Hidalgo y Flores 1992), ha sido sustituida en años recientes por una más sutil, pero basada todavía en la visión patriarcal de que el trabajo sexual es intrínsecamente malo porque se hace con la parte "sucia" del cuerpo. Esta es la etiqueta de la trabajadora sexual como "transmisora de enfermedades", "víctima de explotación", "sobreviviente de violencia", "sujeto de trata de blancas" o "prostituta infantil", etiqueta que se aplica de forma general independientemente de que calce o no con la realidad. Esta visión fundamentalista y patriarcal ha sido ampliamente apoyada y exportada por la Administración Bush en los EEUU (Ashbee 2007) y por otras administraciones previas, siendo favorablemente recibida en Costa Rica por diversos gobiernos, personal de las áreas de sicología y trabajo social, los grupos religiosos y los medios de comunicación (Hidalgo y Flores 1992).

En este estudio, nos apartamos de la visión patriarcal subyacente para aprovechar las ventajas del enfoque científico evolucionista, con su capacidad predictiva y presentación de predicciones concretas que permiten desecharlo si no se cumplen en la realidad, lo cual sería deseable que se aplicara también a otros modelos, como el de la sociología tradicional. En Costa Rica no se ha publicado ningún estudio sociobiológico sobre el trabajo sexual. Nuestro objetivo es presentar el primer análisis del trabajo sexual femenino en el centro de la ciudad de San José, Costa Rica, desde el punto de vista de la sociobiología contemporánea.

\section{METODOLOGÍA}

En 2008 entrevistamos a 78 mujeres que realizan trabajo sexual en la ciudad de San José, capital de Costa Rica. Como no todas las mujeres respondieron todas las preguntas, en los diversos artículos de esta serie la cantidad total de respuestas puede ser menor a 78 . Hubo tres grupos: el grupo 1 corresponde a trabajadoras ambulantes, mujeres que trabajan en la zona aledaña al 
Mercado Borbón y Mercado Central, con tarifa entre US\$3 y US\$8 por hora y edades de 26 a 81 años con muy bajo nivel educativo y apariencia menos atractiva que en los otros grupos; en el grupo 2 están las trabajadoras de dos salas de masajes de San Juan de Tibás (US\$18 a US\$72 por hora y 18 a 33 años, con nivel educativo medio y apariencia intermedia; y en el grupo 3, trabajadoras independientes o VIP (US\$100 la hora; 19 a 38 años con mayor nivel educativo y apariencia más atractiva que en los otros grupos). Las entrevistas se hicieron con base en un cuestionario, se pagó a las entrevistadas su tiempo y los datos se analizaron con el programa estadístico SPSS. Los detalles se presentan en Rojas et al. (2009).

\section{RESULTADOS}

\section{Hombres}

Aunque las edades de los clientes van desde los 15 hasta los 90 años, la mayoría se encuentra en la edad en que los hombres tienen recursos que ofrecer pues ya tienen un empleo $u$ otra fuente de entrada económica (2955 años de edad, Cuadro 1), además de capacidad para realizar el acto sexual. Los adolescentes y los jóvenes, aunque también tienen capacidad sexual, pero apenas están estableciéndose económicamente, no son clientes comunes (Cuadro 1). Los clientes suelen pertenecer a una clase media alta o superior, que puede pagar las tarifas que van desde los US\$13 hasta los US\$100 por hora.

Predominan los clientes casados y en el caso de las trabajadoras de salón, los costarricenses. En las trabajadoras de calle predominan los trabajadores inmigrantes y en las VIP, los provenientes de países industrializados.

\section{CUADRO 1}

Valores promedio de edad de los clientes y tarifas por servicio, para trabajadoras sexuales del centro de la ciudad de San José, Costa Rica

\begin{tabular}{lccc}
\hline Valores & $\begin{array}{c}\text { Grupo1 } \\
\text { Trabajadoras } \\
\text { ambulantes }\end{array}$ & $\begin{array}{c}\text { Grupo 2 } \\
\text { Trabajadoras } \\
\text { de salón }\end{array}$ & $\begin{array}{c}\text { Grupo 3 } \\
\text { Trabajadoras } \\
\text { “VIP" }\end{array}$ \\
\hline $\begin{array}{l}\text { Edad min, } \\
\text { clientes }\end{array}$ & 32,09 & 28,90 & 36,64 \\
$\begin{array}{l}\text { Edad max. } \\
\text { clientes }\end{array}$ & 54,63 & 54,63 & 51,44 \\
$\begin{array}{l}\text { Tarifa por } \\
\text { servicio* }\end{array}$ & US\$13,09 & US\$33,39 & US\$100,32 \\
\hline
\end{tabular}

* Diferencia entre grupos significativa al 0,01 \% (ANDEVA de Kruskal-Wallis)
En general, los servicios más solicitados por los clientes es el sexo oral y el coito normal. Aunque en su mayoría ellas rechazan el servicio cuando los clientes piden acciones que no desean realizar, algunas trabajadoras de salón ofrecen servicios especiales como el sexo anal a cambio de un pago adicional que suele ser de US\$20.

Las relaciones afectivas de amistad entre los clientes y las trabajadoras son frecuentes y en algunos casos se establecen noviazgos, pero poco menos de la mitad de las mujeres entrevistadas también mencionan haber sufrido al menos una vez agresión verbal o física. Aunque no consultamos en nuestro instrumento la frecuencia de esa agresión, las entrevistas abiertas indican que es muy poco frecuente.

\section{Mujeres}

Según nuestras observaciones de campo, el cuidado personal y el arreglo permiten que las trabajadoras se mantengan en el negocio incluso después de la tercera década de edad. Algunas llegan a los 50 años (edades medias en el Cuadro 2), pero, según nuestra opinión, aún se ven atractivas. Las VIP usan perfumes finos y gastan sumas importantes en el cuidado personal. Las ambulantes también pueden seguir trabajando aún a edades mayores y con deterioro físico (sobre todo por la adicción a drogas), pero no por verse atractivas sino porque los clientes que atienden, que incluyen inmigrantes ilegales de Nicaragua, no disponen de recursos económicos suficientes para exigir altos niveles de belleza, juventud y educación.

La gran mayoría de las trabajadoras tienen 1-4 hijas e hijos que mantener (Cuadro 2) y en general no reciben ayuda económica de los respectivos padres. Las ambulantes, que son de mayor edad y menor nivel educativo, tienen más hijos que el resto ( $r s=0.5416 ; p=0.000014)$, en concordancia con la tendencia nacional y generacional hacia una disminución en el número de hijos. Tal vez se arriesgan más a los embarazos porque afirman aceptar ocasionalmente relaciones sin preservativo para no perder a un cliente que pide ese servicio. Los ingresos semanales son mínimos en las trabajadoras ambulantes y máximos en las VIP (Cuadro 2).

Del subgrupo de 58 entrevistadas que respondieron a la pregunta sobre si tenían otras entradas económicas, 48 se dedican exclusivamente al trabajo sexual. Sólo 10 lo combinan con otras actividades (empleadas domésticas, recolección de desechos para reciclaje).

La mitad de las entrevistadas considera que las actividades que realizan representan un trabajo como cualquier otro, excepto en el grupo VIP, formado por mujeres que perciben esta actividad como temporal, mientras logran construir un proyecto de vida distinto, 


\section{CUADRO 2}

Valores promedio para edad, número de hijos, clientes e ingresos en trabajadoras sexuales del centro de la ciudad de San José, Costa Rica

\begin{tabular}{|c|c|c|c|}
\hline Valores & $\begin{array}{c}\text { Grupo } 1 \\
\text { Trabajadoras } \\
\text { ambulantes }\end{array}$ & $\begin{array}{c}\text { Grupo } 2 \\
\text { Trabajadoras } \\
\text { de salón }\end{array}$ & $\begin{array}{c}\text { Grupo } 3 \\
\text { Trabajadoras } \\
\text { "VIP" }\end{array}$ \\
\hline Edad $^{* *}$ & 41.21 & 24.33 & 25.94 \\
\hline No. Hijos** & 3.78 & 1.28 & 1.18 \\
\hline $\begin{array}{l}\text { Clientes } \\
\text { semana } \\
\text { buena** }\end{array}$ & 9.63 & 28.50 & 13.88 \\
\hline $\begin{array}{l}\text { Clientes } \\
\text { semana } \\
\text { mala* }\end{array}$ & 3.87 & 8.28 & 4.88 \\
\hline $\begin{array}{l}\text { Ingresos } \\
\text { semanales** }\end{array}$ & US\$ 52.68 & US\$ 250.00 & US\$ 531.05 \\
\hline
\end{tabular}

* Diferencia entre grupos significativa al 0,05 \% (ANDEVA de KruskalWallis)

** Diferencia entre grupos significativa al 0,01 \% (ANDEVA de KruskalWallis)

pues unas lo describen como pecado y otras como socialmente incorrecto.

En general, reciben ingresos muy superiores a los que ganarían en empleos acordes con su nivel educativo, como conserje o recepcionista (US\$109,93 y US\$119,39 semanales respectivamente).

Prácticamente la totalidad de las trabajadoras (99\%) administra el dinero que gana: no se da la explotación por parte de un "mantenido" común en los países donde el trabajo sexual es ilegal. En su mayoría, el dinero obtenido en este trabajo lo destinan a las necesidades básicas de la familia; una pequeña proporción de las trabajadoras (18\%), principalmente en el grupo VIP, destinan parte importante de sus ingresos a los cuidados personales. Pocas (5.6\%) destinan ingresos a la recreación y la formación profesional. En todos los grupos hay una proporción $(60,7 \%)$ que ahorra, y las que ganan más ahorran más.

\section{DISCUSIÓN}

A la pregunta de por qué una mujer acepta tener relaciones sexuales con un hombre, a menudo de forma exclusiva y prolongada, la psicología pre-evolutiva respondía que lo hacía por hallar en él similitud o complementariedad (Buss y Shackelford 2008). El trabajo sexual solía explicarse como resultado de traumas en la niñez, entre otras ideas (Buss 2009). La sociobiología y la sicología evolutiva dan una explicación más compleja: debido a la diferencia sexual en cuanto a la inversión que obligatoriamente se hace en la prole, la mujer es altamente selectiva y busca compromiso en la provisión de recursos suficientes, ya que normalmente permanece muchos años dedicada a sus hijas e hijos. El hombre es menos selectivo, por lo que se siente atraído a tener intimidad sexual con mayor cantidad de parejas que la mujer. Además, cuando prevee un compromiso, el hombre busca claves físicas de fertilidad y claves mentales de habilidad para criar a la prole (Buss y Shackelford 2008, Buss 2009).

Por su naturaleza científica, el modelo evolucionista aplicado al trabajo sexual humano hace predicciones claras que permiten desecharlo si no se cumplen. En el caso del trabajo sexual, el modelo afirma que el trabajo sexual será una estrategia en que las mujeres en edad reproductiva y con necesidad de recursos, obtienen estos recursos de algunos hombres, aprovechando la tendencia masculina al apareamiento múltiple durante la edad reproductiva (Alcock 2001, Wilson y Wilson 2007). El modelo predice que las mujeres casi siempre cobran por el servicio, y que las más atractivas por su belleza, intelecto y educación ganan más (Alcock 2001, Wilson y Wilson 2007).

Las predicciones se cumplen: los clientes están en edad reproductiva y deben pagar por el servicio incluso en los casos en que establecen una relación sentimental con las trabajadoras. Y en efecto, las mujeres se concentran en su ámbito reproductivo (la mayoría entre 24 y 41 años de edad), el $100 \%$ cobran por sus servicios, incluso si han llegado a tener una relación sentimental con el cliente, y las mujeres con mayor atractivo físico y educación ganan hasta 10 veces más que el resto. El $98 \%$ de ellas carecen de otra fuente de trabajo y el $69 \%$ no tienen pareja que provea dinero para el sostenimiento de los hijos. El otro 31\% tiene pareja pero no recibe suficiente dinero de él.

En conclusión, los datos obtenidos en nuestro estudio con 78 trabajadoras sexuales de la ciudad de San José calzan con las predicciones del modelo evolucionista.

\section{AGRADECIMIENTOS}

Agradecemos a Mónica Chávez por su ayuda como asistente del proyecto; a las mujeres que compartieron sus historias y opiniones. También a La Sala, a los locales y a la Fundación RAHAB; y a la Vicerrectoría de Investigación de la UNED por el financiamiento; así como a Katya Calderón, sin cuyo eficaz apoyo habría sido imposible concluir este estudio. Dedicamos nuestro artículo a las trabajadoras sexuales: pese a los prejuicios patriarcales, deberían recibir la misma comprensión y el mismo respeto que cualquier otro ser humano al que se le permite elegir su 
modo de ganarse el sustento y se le otorgan las condiciones necesarias para hacerlo dignamente.

\section{RESUMEN}

En Costa Rica, el trabajo sexual femenino ha sido estudiado durante décadas según una visión pre-evolucionista y a menudo, subyacentemente patriarcal pues el trabajo sexual es visto como una patología social, ignorando todos los avances hechos en este campo por la sociobiología y al sicología evolutiva. En este estudio, medimos el cumplimiento de las predicciones del modelo evolucionista con base en 78 mujeres que realizan trabajo sexual en la ciudad de San José, capital de Costa Rica, entrevistadas en 2008, con edades entre 18 y 81 años. Las entrevistas se hicieron con base en un cuestionario, se pagó a las entrevistadas su tiempo y los datos se analizaron con el programa estadístico SPSS. Todas las predicciones del modelo evolutivo se cumplen en esta población: los clientes son predominantemente hombres en edad reproductiva (29 a 54 años) que pagan a las mujeres por su actividad sexual incluso en los casos en que establecen una relación sentimental con las trabajadoras. Las predicciones del modelo sobre las trabajadoras también se cumplen, pues son primordialmente mujeres en edad reproductiva (24 a 41 años), el $100 \%$ cobran por sus servicios y las mujeres más atractivas por su belleza, intelecto y educación ganan hasta 10 veces más que el resto. Debido a que en la especie humana se da intercambio de recursos por servicios sexuales, el $98 \%$ de las trabajadoras sexuales son mujeres que carecen de otra fuente de trabajo y el $69 \%$ no tienen pareja que provea dinero para el sostenimiento de los hijos. El otro $31 \%$ tiene pareja pero no recibe suficiente dinero de él.

\section{PALABRAS CLAVE}

Evolución del comportamiento sexual humano, trabajo sexual, Costa Rica, sociobiología, sicología evolutiva, prostitución, recursos por servicios sexuales, patriarcado.

\section{REFERENCIAS}

Alcock, J. 2001. The Triumph of Sociobiology. Oxford University Press, Nueva York, EEUU.

Ashbee, E. 2007. The Bush Administration, Sex and the Moral Agenda. Manchester University, Manchester, Inglaterra.

Barkow, J.H. (Ed.) 2005. Missing the Revolution: Darwinism for Social Scientists. Oxford University, Nueva York, Nueva York, EEUU.

Bouissac, P. 2006. Hoarding behavior: A better evolutionary account of money psychology? Behavioral and Brain Sciences 29: 181-182.

Buss, D.M. 2009. The Great Struggles of Life: Darwin and the Emergence of Evolutionary Psychology. American Psychologist 64: 140-148.

Buss, D.M. \& T.K. Shackelford. 2008. Attractive women want it all: Good genes, economic investment, parenting proclivities, and emotional commitment. Evolutionary Psychology 6: 134-146.
Farley, M. 2005. Prostitution harms women even if indoors. Violence Against Women 11: 950-964.

Foster, K.R., K. Parkinson \& C.R. Thompson. 2007. What can microbial genetics teach sociobiology? Trends in Genetics 23: $74-80$.

Geary, D.C. 2006. Sexual selection and the evolution of human sex differences. Psychological Topics 15: 203-238.

Herrera, M. 2000. Detrás del telón... Entre lo imaginario y lo real. Estudio cualitativo sobre mujeres en prostitución. Tesis de Licenciatura, Escuela de Trabajo Social, Universidad de Costa Rica, San José, Costa Rica.

Hidalgo, R. \& M. Flores. 1992. El autoritarismo en la vida cotidiana: SIDA, homofobia y moral sexual. Revista de Ciencias Sociales UCR 98: 35-44.

Lagarde, M. 2003. El feminismo y la mirada entre mujeres. Ponencia en el Seminario Internacional sobre Liderazgo y Dirección para mujeres. "Poder y empoderamiento de las mujeres". Valencia, España.

Leigh, C. 2008. The strange relationship between feminism and sex work. On The Issues Magazine (9 de noviembre 2008). (Disponible en http://www.alternet.org/story/98645/; consultado el 11 de noviembre de 2008)

López, M. 1992. Costa Rica: la opinión pública y el SIDA. Ciencias Sociales 58: 55-64.

Méndez, Z. 1992. Socialización y estereotipos sexuales en Costa Rica. Ciencias Sociales 39: 29-45.

Miller, B., J. Tybur \& B. Jordan. 2007. Ovulatory cycle effects on tip earnings by lap dancers: economic evidence for human estrus? Evolution and Human Behavior 28: 375-381.

Murphy, E. 1998. Historia de los grandes burdeles del mundo. Temas de Hoy, Madrid, España.

Rojas, A. \& M. Scott. 1994. Relatos de vida y representación del dinero en cinco mujeres prostitutas del sector central de San José. Tesis de Licenciatura, Escuela de Psicología, Universidad de Costa Rica, San José, Costa Rica.

Rojas C., R., J. Monge-Nájera, I. Ramírez S. \& R. Morales B. 2009. El mercado del trabajo sexual femenino en la ciudad de San José, Costa Rica. Cuadernos de Investigación UNED 1: $13-25$

Saad, G. 2007. The evolutionary bases of consumption. Lawrence Eribaum, Nueva York, Nueva York, EEUU.

Schiebinger, I. 1993. Nature's body. Gender in the making of modern science. Beakon Press, Boston, Massachussetts, EEUU.

Wilson, D.S. \& E.O. Wilson. 2007. Rethinking the theoretical foundation of sociobiology. The Quaterly Review of Biology 82: $327-348$.

Este artículo fue editado por Zaidett Barrientos 
